\title{
Invariant Inhomogeneous Bianchi Type-I Cosmological Models with Electromagnetic Fields Using Lie Group Analysis in Lyra Geometry
}

\author{
Ahmad T. Ali ${ }^{1,2}$ \\ ${ }^{1}$ Department of Mathematics, Faculty of Science, King Abdulaziz University, P.O. Box 80203, Jeddah 21589, Saudi Arabia \\ ${ }^{2}$ Mathematics Department, Faculty of Science, Al-Azhar University, Nasr City, Cairo 11884, Egypt
}

Correspondence should be addressed to Ahmad T. Ali; atali71@yahoo.com

Received 28 December 2013; Accepted 3 June 2014; Published 19 June 2014

Academic Editor: Maria Bruzón

Copyright (C) 2014 Ahmad T. Ali. This is an open access article distributed under the Creative Commons Attribution License, which permits unrestricted use, distribution, and reproduction in any medium, provided the original work is properly cited.

\begin{abstract}
We find a new class of invariant inhomogeneous Bianchi type-I cosmological models in electromagnetic field with variable magnetic permeability. For this, Lie group analysis method is used to identify the generators that leave the given system of nonlinear partial differential equations (NLPDEs) (Einstein field equations) invariant. With the help of canonical variables associated with these generators, the assigned system of PDEs is reduced to ordinary differential equations (ODEs) whose simple solutions provide nontrivial solutions of the original system. A new class of exact (invariant-similarity) solutions have been obtained by considering the potentials of metric and displacement field as functions of coordinates $x$ and $t$. We have assumed that $F_{12}$ is only nonvanishing component of electromagnetic field tensor $F_{i j}$. The Maxwell equations show that $F_{12}$ is the function of $x$ alone whereas the magnetic permeability $\bar{\mu}$ is the function of $x$ and $t$ both. The physical behavior of the obtained model is discussed.
\end{abstract}

\section{Introduction}

The inhomogeneous cosmological models play a significant role in understanding some essential features of the universe, such as the formation of galaxies during the early stages of evolution and process of homogenization. Therefore, it will be interesting to study inhomogeneous cosmological models. The best-known inhomogeneous cosmological model is the Lemaitre-Tolman model (or LT model) which deals with the study of structure in the universe by means of exact solutions of Einstein's field equations. Some other known exact solutions of inhomogeneous cosmological models are the Szekeres metric, Szafron metric, Stephani metric, KantowskiSachs metric, Barnes metric, Kustaanheimo-Qvist metric, and Senovilla metric [1].

Einstein's general theory relativity is based on Riemannian geometry. If one modifies the Riemannian geometry, then Einstein's field equations will be changed automatically from its original form. Modifications of Riemannian geometry have developed to solve the problems such as unification of gravitation with electromagnetism, problems arising when the gravitational field is coupled to matter fields, and singularities of standard cosmology. In recent years, there has been considerable interest in alternative theory of gravitation to explain the above-unsolved problems. Long ago, since 1951, Lyra [2] proposed a modification of Riemannian geometry by introducing a gauge function into the structureless manifold that bears a close resemblance to Weyl's geometry.

Using the above modification of Riemannian geometry Sen $[3,4]$ and Sen and Dunn [5] proposed a new scalar tensor theory of gravitation and constructed it very similar to Einstein field equations. Based on Lyra's geometry, the field equations can be written as $[3,4]$

$$
R_{i j}-\frac{1}{2} g_{i j} R+\frac{3}{2} \phi_{i} \phi_{j}-\frac{3}{4} g_{i j} \phi_{k} \phi^{k}=-\chi T_{i j}
$$

where $\phi_{i}$ is the displacement vector and other symbols have their usual meaning as in Riemannian geometry.

Halford [6] has argued that the nature of constant displacement field $\phi_{i}$ in Lyra's geometry is very similar to cosmological constant $\Lambda$ in the normal general relativistic 
theory. Halford also predicted that the present theory will provide the same effects within observational limits, as far as the classical solar system tests are concerned, as well as tests based on the linearized form of field equations. For a review on Lyra geometry, one can see [7].

Recently, Pradhan et al. [8-14], Casana et al. [15], Rahaman et al. [16], Bali and Chandnani [17, 18], Kumar and Singh [19], Yadav et al. [20], Rao et al. [21], Zia and Singh [22], and Ali and Rahaman [23] have studied cosmological models based on Lyra's geometry in various contexts.

To study the nonlinear physical phenomena [24-27], it is important to search the exact solutions of nonlinear PDEs. Ovsiannikov [28] is the pioneer who had observed that the usual Lie infinitesimal invariance approach could as well be employed in order to construct symmetry groups [2931]. The symmetry groups of a differential equation could be defined as the groups of continuous transformations that lead a given family of equations invariant [32-35] and are proved to be important to solve the nonlinear equations of the models to describe complex physical phenomena in various fields of science, especially in fluid mechanics, solid state physics, plasma physics, plasma wave, and general relativity.

In this paper, we have obtained exact solutions of Einstein's modified field equations in inhomogeneous space-time Bianchi type-I cosmological model within the frame work of Lyra's geometry in the presence of magnetic field with variable magnetic permeability and time varying displacement vector $\beta(x, t)$ using the so-called symmetry analysis method. Since the field equations are highly nonlinear differential equations, therefore symmetry analysis method can be successfully applied to nonlinear differential equations. The similarity (invariant) solutions help to reduce the independent variables of the problem, and therefore we employ this method in the investigation of exact solution of the field equations. In general, invariant solutions will transform the system of nonlinear PDEs into a system of ODEs. We attempted to find a new class of exact (invariant) solutions for the field equations based on Lyra geometry.

The scheme of the paper is as follows. Magnetized inhomogeneous Bianchi type-I cosmological model with variable magnetic permeability based on Lyra geometry is introduced in Section 2. In Section 3, we have performed symmetry analysis and have obtained isovector fields for Einstein field equations under consideration. In Section 4, we found new class of exact (invariant) solutions for Einstein field equations. Section 5 is devoted to study of some physical and geometrical properties of the model.

\section{The Metric and Field Equations}

We consider Bianchi type-I metric, with the convention $\left(x^{0}=\right.$ $\left.t, x^{1}=x, x^{2}=y, x^{3}=z\right)$, in the form

$$
d s^{2}=d t^{2}-A^{2} d x^{2}-B^{2} d y^{2}-C^{2} d z^{2},
$$

where $A$ is a function of $t$ only while $B$ and $C$ are functions of $x$ and $t$. Without loss of generality, we can put the following transformation:

$$
B=A f, \quad C=A g
$$

where $f$ and $g$ are functions of $x$ and $t$. The volume element of model (2) is given by

$$
V=\sqrt{-g}=A^{3} f g .
$$

The four-acceleration vector, the rotation, the expansion scalar, and the shear scalar characterizing the four-velocity vector field, $u^{i}$, satisfying the relation in comoving coordinate system

$$
g_{i j} u^{i} u^{j}=1, \quad u^{i}=u_{i}=(1,0,0,0)
$$

respectively, have the usual definitions as given by Raychaudhuri [36]

$$
\begin{aligned}
& \dot{u}_{i}=u_{i ; j} u^{j}, \\
& \omega_{i j}=u_{[i ; j]}+\dot{u}_{[i} u_{j]}, \\
& \Theta=u_{; i}^{i}, \\
& \sigma^{2}=\frac{1}{2} \sigma_{i j} \sigma^{i j},
\end{aligned}
$$

where

$$
\sigma_{i j}=u_{(i ; j)}+\dot{u}_{(i} u_{j)}-\frac{1}{3} \Theta\left(g_{i j}+u_{i} u_{j}\right) .
$$

In view of metric (2), the four-acceleration vector, the rotation, the expansion scalar, and the shear scalar given by (6) can be written in a comoving coordinates system as

$$
\begin{aligned}
\dot{u}_{i}= & 0, \\
\omega_{i j}= & 0, \\
\Theta= & \frac{3 \dot{A}}{A}+\frac{f_{t}}{f}+\frac{g_{t}}{g}, \\
\sigma^{2}= & \frac{\dot{A}}{A}\left(\frac{2 \dot{A}}{A}+\frac{4 f_{t}}{3 f}+\frac{4 g_{t}}{3 g}\right)+\frac{5 f_{t}^{2}}{9 f^{2}} \\
& +\frac{f_{t} g_{t}}{9 f g}+\frac{5 g_{t}^{2}}{9 g^{2}},
\end{aligned}
$$

where the nonvanishing components of the shear tensor $\sigma_{i}^{j}$ are

$$
\begin{array}{ll}
\sigma_{1}^{1}=-\frac{f_{t}}{3 f}-\frac{g_{t}}{3 f}, & \sigma_{2}^{2}=\frac{2 f_{t}}{3 f}-\frac{g_{t}}{3 g}, \\
\sigma_{3}^{3}=\frac{2 g_{t}}{3 g}-\frac{f_{t}}{3 f}, & \sigma_{4}^{4}=-\frac{2 \dot{A}}{A}-\frac{2 f_{t}}{3 f}-\frac{2 g_{t}}{3 g} .
\end{array}
$$

To study the cosmological model, we use the field equations in Lyra geometry given in (1) in which the displacement field vector $\phi_{i}$ is given by

$$
\phi_{i}=(\beta(x, t), 0,0,0) .
$$


$T_{i j}$ is the energy momentum tensor given by

$$
T_{i j}=(\rho+p) u_{i} u_{j}-p g_{i j}+E_{i j}
$$

where $E_{i j}$ is the electromagnetic field given by Lichnerowicz [37]:

$$
E_{i j}=\bar{\mu}\left[h_{l} h^{l}\left(u_{i} u_{j}-\frac{1}{2} g_{i j}\right)+h_{i} h_{j}\right] .
$$

Here $\rho$ and $p$ are the energy density and isotropic pressure, respectively, while $\bar{\mu}$ is the magnetic permeability and $h_{i}$ is the magnetic flux vector defined by

$$
h_{i}=\frac{\sqrt{-g}}{2 \bar{\mu}} \epsilon_{i j k l} F^{k l} u^{j} \text {. }
$$

$F_{i j}$ is the electromagnetic field tensor and $\epsilon_{i j k l}$ is a Levi-Civita tensor density. If we consider the current flow along $z$-axis, then $F_{12}$ is only nonvanishing component of $F_{i j}$. Then the Maxwell equations

$$
\begin{gathered}
F_{i j ; k}+F_{j k ; i}+F_{k i ; j}=0, \\
{\left[\frac{1}{\bar{\mu}} F^{i j}\right]_{; j}=J^{i}}
\end{gathered}
$$

require $F_{12}$ to be function of $x$ alone [38]. We assume the magnetic permeability as a function of both $x$ and $t$. Here the semicolon represents a covariant differentiation.

For the line element (2), field equation (1) can be reduced to the following system of NLPDEs:

$$
\begin{gathered}
E_{1}=\frac{f_{x t}}{f}+\frac{g_{x t}}{g}=0, \\
E_{2}=\frac{f_{t t}}{f}+\frac{f_{t} g_{t}}{f g}+\frac{1}{A^{2}}\left(\frac{g_{x x}}{g}-\frac{f_{x} g_{x}}{f g}\right)+\frac{3 \dot{A} f_{t}}{A f}=0 \\
\chi \rho+\frac{3}{4} \beta^{2}=\frac{g_{t t}}{2 g}+\frac{3 f_{t} g_{t}}{2 f g}-\frac{1}{A^{2}}\left(\frac{f_{x x}}{2 f}+\frac{g_{x x}}{g}+\frac{3 f_{x} g_{x}}{2 f g}\right) \\
+\frac{\dot{A}}{A}\left(\frac{2 f_{t}}{f}+\frac{g_{t}}{2 g}+\frac{3 \dot{A}}{A}\right), \\
\chi p+\frac{3}{4} \beta^{2}=\frac{1}{2 A^{2}}\left(\frac{f_{x x}}{f}+\frac{f_{x} g_{x}}{f g}\right)-\frac{f_{t t}}{f}-\frac{g_{t t}}{2 g}-\frac{f_{t} g_{t}}{2 f g} \\
-\frac{\dot{A}}{A}\left(\frac{3 f_{t}}{f}+\frac{\dot{A}}{A}\right)-\frac{2 \ddot{A}}{A}, \\
\frac{\chi F_{12}^{2}}{\bar{\mu} A^{4} f^{2}}=\frac{1}{A^{2}}\left(\frac{f_{x x}}{f}-\frac{f_{x} g_{x}}{f g}\right)+\frac{g_{t t}}{g}+\frac{f_{t} g_{t}}{f g}+\frac{3 \dot{A} g_{t}}{A g} .
\end{gathered}
$$

\section{Symmetry Analysis Method}

Equations (15)-(16) are highly nonlinear PDEs and hence it is so difficult to handle since there exist no standard methods for obtaining analytical solution. The system (15) is nonlinear
PDEs of second order for the two unknowns $f$ and $g$. If we solve this system, then we can get the solution of the field equations. In order to obtain an exact solution of the system of nonlinear PDEs (15), we will use the symmetry analysis method. For this we write

$$
\begin{aligned}
& x_{i}^{*}=x_{i}+\epsilon \xi_{i}\left(x_{j}, u_{\beta}\right)+\mathbf{o}\left(\epsilon^{2}\right), \\
& u_{\alpha}^{*}=u_{\alpha}+\epsilon \eta_{\alpha}\left(x_{j}, u_{\beta}\right)+\mathbf{o}\left(\epsilon^{2}\right),
\end{aligned} \quad i, j, \alpha, \beta=1,2,
$$

as the infinitesimal Lie point transformations. We have assumed that the system (15) is invariant under the transformations given in (17). The corresponding infinitesimal generator of Lie groups (symmetries) is given by

$$
X=\sum_{i=1}^{2} \xi_{i} \frac{\partial}{\partial x_{i}}+\sum_{\alpha=1}^{2} \eta_{\alpha} \frac{\partial}{\partial u_{\alpha}},
$$

where $x_{1}=x, x_{2}=t, u_{1}=f$, and $u_{2}=g$. The coefficients $\xi_{1}, \xi_{2}, \eta_{1}$, and $\eta_{2}$ are the functions of $x, t, f$, and $g$. These coefficients are the components of infinitesimals symmetries corresponding to $x, t, f$, and $g$, respectively, to be determined from the invariance conditions:

$$
\left.\operatorname{Pr}^{(2)} X\left(E_{m}\right)\right|_{E_{m}=0}=0,
$$

where $E_{m}=0, m=1,2$ are the system (15) under study and $\operatorname{Pr}^{(2)}$ is the second prolongation of the symmetries $X$. Since our equations (15) are at most of order two, therefore, we need second order prolongation of the infinitesimal generator in (19). It is worth noting that the 2 nd order prolongation is given by

$$
\operatorname{Pr}^{(2)} X=X+\sum_{i=1}^{2} \sum_{\alpha=1}^{2} \eta_{\alpha, i} \frac{\partial}{\partial u_{\alpha, i}}+\sum_{j=1}^{2} \sum_{i=1}^{2} \sum_{\alpha=1}^{2} \eta_{\alpha, i j} \frac{\partial}{\partial u_{\alpha, i j}},
$$

where

$$
\begin{aligned}
& \eta_{\alpha, i}=D_{i}\left[\eta_{\alpha}-\sum_{j=1}^{2} \xi_{j} u_{\alpha, j}\right]+\sum_{j=1}^{2} \xi_{j} u_{\alpha, i j}, \\
& \eta_{\alpha, i j}=D_{i j}\left[\eta_{\alpha}-\sum_{k=1}^{2} \xi_{k} u_{\alpha, k}\right]+\sum_{k=1}^{2} \xi_{k} u_{\alpha, i j k} .
\end{aligned}
$$

The operator $D_{i}\left(D_{i j}\right)$ is called the total derivative (Hach operator) and takes the following form:

$$
D_{i}=\frac{\partial}{\partial x_{i}}+\sum_{\alpha=1}^{2} u_{\alpha, i} \frac{\partial}{\partial u_{\alpha}}+\sum_{j=1}^{2} \sum_{\alpha=1}^{2} u_{\alpha, i j} \frac{\partial}{\partial u_{\alpha, j}},
$$

where $D_{i j}=D_{i}\left(D_{j}\right)=D_{j}\left(D_{i}\right)=D_{j i}$ and $u_{\alpha, i}=\partial u_{\alpha} / \partial x_{i}$.

Expanding the system (19) with the aid of Mathematica program, along with the original system (15) to eliminate $f_{x t}$ and $g_{x x}$ while we set the coefficients involving $f_{x}, f_{t}, f_{x x}, f_{t t}$, $g_{x}, g_{t}, g_{x t}$, and $g_{t t}$ and various products equal zero, these gives rise to the essential set of overdetermined equations. Solving the set of these determining equations, the components of symmetries take the following form:

$$
\begin{gathered}
\xi_{1}=c_{1} x+c_{2}, \quad \xi_{2}=c_{3} t+c_{4}, \\
\eta_{1}=0, \quad \eta_{2}=c_{5} g,
\end{gathered}
$$


where $c_{i}, i=1,2, \ldots, 5$, are arbitrary constants and the function $A(t)$ must equal

$$
A(t)=c_{6} \xi_{2}(t) \exp \left[-c_{1} \int \frac{d t}{\xi_{2}(t)}\right]
$$

Therefore, $A(t)$ becomes

$$
\begin{aligned}
& A(t)=c_{6}\left(c_{3} t+c_{4}\right)^{1-\left(c_{1} / c_{3}\right)}, \quad \text { if } c_{3} \neq 0, \\
& A(t)=c_{7} \exp \left[-\frac{c_{1}}{c_{4}} t\right], \quad \text { if } c_{3}=0,
\end{aligned}
$$

where $c_{6}$ and $c_{7}=c_{4} c_{6}$ are arbitrary constants.

\section{Invariant Solutions}

The characteristic equations corresponding to the symmetries (23) are given by

$$
\frac{d x}{c_{1} x+c_{2}}=\frac{d t}{c_{3} t+c_{4}}=\frac{d f}{0}=\frac{d g}{c_{5} g} .
$$

By solving the above system, we have the following four cases.

Case 1 . When $c_{1} \neq 0$ and $c_{3} \neq 0$, the similarity variable and similarity functions can be written as follows:

$$
\begin{gathered}
\xi=\frac{x+a}{(t+b)^{c}}, \quad f(x, t)=\Psi(\xi), \\
g(x, t)=(x+a)^{d} \Phi(\xi),
\end{gathered}
$$

where $a=c_{2} / c_{1}, b=c_{4} / c_{3}, c=c_{1} / c_{3}$, and $d=c_{5} / c_{1}$ are arbitrary constants. In this case, $A(t)=q(t+b)^{1-c}$, where $q=c_{6} c_{3}^{1-c}$. Substituting the transformations (27) in the field (15) leads to the following system of ODEs:

$$
\begin{gathered}
\frac{\xi \Psi^{\prime \prime}+\Psi^{\prime}}{\Psi}+\frac{\xi \Phi^{\prime \prime}+(1+d) \Phi^{\prime}}{\Phi}=0 \\
\frac{\left(c^{2} q^{2} \xi^{2}-1\right) \xi \Phi^{\prime} \Psi^{\prime}}{\Phi \Psi}+\frac{2 d \Phi^{\prime}+\xi \Phi^{\prime \prime}}{\Phi} \\
+\frac{c^{2} q^{2} \xi^{3} \Psi^{\prime \prime}-\left[d+2 c(1-2 c) q^{2} \xi^{2}\right] \Psi^{\prime}}{\Psi}=\frac{d(1-d)}{\xi} .
\end{gathered}
$$

If one solves the system of second order NLPDEs (28)-(29), one can obtain the exact solutions of the original Einstein field equations (15) corresponding to reduction (30).

Case 2. When $c_{1} \neq 0$ and $c_{3}=0$, the similarity variable and similarity functions can be written as follows:

$$
\begin{gathered}
\xi=(x+a) \exp [b t], \quad f(x, t)=\Psi(\xi), \\
g(x, t)=(x+a)^{c} \Phi(\xi),
\end{gathered}
$$

where $a=c_{2} / c_{1}, b=-c_{1} / c_{4}$, and $c=c_{5} / c_{1}$ are arbitrary constants. In this case, $A(t)=d \exp [b t]$, where $d=c_{7}$.
Substituting transformations (30) in the field equations (15) leads to the following system of ODEs:

$$
\begin{gathered}
\frac{\xi \Psi^{\prime \prime}+\Psi^{\prime}}{\Psi}+\frac{\xi \Phi^{\prime \prime}+(1+c) \Phi^{\prime}}{\Phi}=0, \\
\frac{\left(b^{2} d^{2} \xi^{2}-1\right) \xi \Phi^{\prime} \Psi^{\prime}}{\Phi \Psi}+\frac{2 c \Phi^{\prime}+\xi \Phi^{\prime \prime}}{\Phi} \\
+\frac{b^{2} d^{2} \xi^{3} \Psi^{\prime \prime}+\left(4 b^{2} d^{2} \xi^{2}-c\right) \Psi^{\prime}}{\Psi}=\frac{c(1-c)}{\xi} .
\end{gathered}
$$

If one solves the system of second order NLPDEs (31)-(32), one can obtain the exact solutions of the original Einstein field equations (15) corresponding to reduction (30).

Case 3. When $c_{1}=0$ and $c_{3} \neq 0$, the similarity variable and similarity functions can be written as follows:

$$
\begin{gathered}
\xi=\frac{\exp [a x]}{t+b}, \quad f(x, t)=\Psi(\xi), \\
g(x, t)=\Phi(\xi) \exp [a x],
\end{gathered}
$$

where $a=c_{3} / c_{2}$ and $b=c_{4} / a_{3}$ are arbitrary constants. In this case we have $A(t)=c(t+b)$, where $c=c_{6}$. Substituting the transformations (33) in the field equations (15) leads to the following system of ODEs:

$$
\begin{gathered}
\frac{\xi \Psi^{\prime \prime}+\Psi^{\prime}}{\Psi}+\frac{\xi \Phi^{\prime \prime}+2 \Phi^{\prime}}{\Phi}=0, \\
\xi\left[\frac{\left(c^{2}-a^{2}\right) \xi \Phi^{\prime} \Psi^{\prime}}{\Phi \Psi}+\frac{a^{2}\left(3 \Phi^{\prime}+\xi \Phi^{\prime \prime}\right)}{\Phi}\right. \\
\left.+\frac{c^{2} \xi \Psi^{\prime \prime}-\left(c^{2}+a^{2}\right) \Psi^{\prime}}{\Psi}\right]+a^{2}=0 .
\end{gathered}
$$

Without loss of generality, we can take the following useful transformation:

$$
\begin{gathered}
\xi=\exp [\theta], \quad \frac{d \Psi}{d \xi}=\exp [-\theta] \frac{d \Psi}{d \theta}, \\
\frac{d^{2} \Psi}{d \xi^{2}}=\exp [-2 \theta]\left(\frac{d^{2} \Psi}{d \theta^{2}}-\frac{d \Psi}{d \theta}\right) .
\end{gathered}
$$

Then the system of ODEs (34) transforms to

$$
\begin{gathered}
\frac{\ddot{\Psi}}{\Psi}+\frac{\ddot{\Phi}+\dot{\Phi}}{\Phi}=0, \\
\frac{\left(c^{2}-a^{2}\right) \dot{\Phi} \dot{\Psi}}{\Phi \Psi}+\frac{a^{2}(2 \dot{\Phi}+\ddot{\Phi})}{\Phi} \\
+\frac{c^{2} \ddot{\Psi}-\left(2 c^{2}+a^{2}\right) \dot{\Psi}}{\Psi}+a^{2}=0 .
\end{gathered}
$$

Equation (36) can be written in the following form:

$$
\ddot{\Psi}=-\frac{\Psi}{\Phi}(\ddot{\Phi}+\dot{\Phi}) \text {. }
$$


From the above equation, if we substitute $\ddot{\Psi}$ in (37), we can obtain the following form:

$$
\begin{gathered}
\left(c^{2}-a^{2}\right)\left[\frac{\dot{\Phi} \dot{\Psi}}{\Phi \Psi}-\frac{\ddot{\Phi}}{\Phi}\right]+\left(2 a^{2}-c^{2}\right)\left(\frac{\dot{\Phi}}{\Phi}\right) \\
-\left(2 c^{2}+a^{2}\right)\left(\frac{\dot{\Psi}}{\Psi}\right)+a^{2}=0 .
\end{gathered}
$$

Equation (39) is a nonlinear ODE which is very difficult to solve. However, it is worth noting that this equation is easy to solve when $c=a$. In this case, we can integrate (39) and obtain the following:

$$
\Phi(\theta)=a_{1} \Psi^{3}(\theta) \exp [-\theta]
$$

where $a_{1}$ is an arbitrary constant of integration. Substituting (40) in (36), we have the following ODE of the function $\Psi$ only:

$$
\Psi(4 \ddot{\Psi}-3 \dot{\Psi})+6 \dot{\Psi}^{2}=0
$$

The general solution of the above equation is

$$
\Psi(\theta)=a_{3}\left(a_{2}+\exp \left[\frac{3 \theta}{4}\right]\right)^{2 / 5},
$$

where $a_{2}$ and $a_{3}$ are arbitrary constants of integration. Now, by using (40) and the inverse of transformations (35) and (33), we can find the solution as follows:

$$
\begin{aligned}
A(t) & =a(t+b), \\
f(x, t) & =a_{3}\left(a_{2}+\left[\frac{\exp [a x]}{t+b}\right]^{3 / 4}\right)^{2 / 5}, \\
g(x, t) & =a_{1} a_{3}(t+b)\left(a_{2}+\left[\frac{\exp [a x]}{t+b}\right]^{3 / 4}\right)^{6 / 5} .
\end{aligned}
$$

It is observed from (43) and (3) that the line element (2) can be written in the following form:

$$
\begin{aligned}
d s^{2}= & d t^{2}-a^{2}(t+b)^{2} d x^{2}-d^{2}(t+b)^{2} \\
& \times\left(a_{2}+\left[\frac{\exp [a x]}{t+b}\right]^{3 / 4}\right)^{4 / 5} d y^{2} \\
& -q^{2}(t+b)^{4}\left(a_{2}+\left[\frac{\exp [a x]}{t+b}\right]^{3 / 4}\right)^{12 / 5} d z^{2},
\end{aligned}
$$

where $a, b, d=a a_{3}, q=a a_{1} a_{3}$, and $a_{2}$ are arbitrary constants.

Case 4. When $c_{1}=c_{3}=0$, the similarity variable and similarity functions can be written as follows:

$$
\begin{aligned}
& \xi=a x+b t, \quad f(x, t)=\Psi(\xi), \\
& g(x, t)=\Phi(\xi) \exp [c x],
\end{aligned}
$$

where $a=c_{4}, b=-c_{2}$, and $c=c_{5} / c_{2}$ are arbitrary constants. In this case we have $A(t)=c_{7}$. Substituting transformations (45) in field equations (15) leads to the following system of ODEs:

$$
\begin{gathered}
\frac{a \Psi^{\prime \prime}}{\Psi}+\frac{a \Phi^{\prime \prime}+c \Phi^{\prime}}{\Phi}=0, \\
\frac{\left(b^{2} r^{2}-a^{2}\right) \Phi^{\prime} \Psi^{\prime}}{\Phi \Psi}+\frac{a\left(2 c \Phi^{\prime}+a \Phi^{\prime \prime}\right)}{\Phi} \\
+\frac{b^{2} r^{2} \Psi^{\prime \prime}-a c \Psi^{\prime}}{\Psi}+c^{2}=0 .
\end{gathered}
$$

Equation (46) can be written in the following form:

$$
\Psi^{\prime \prime}=-\frac{\Psi}{a \Phi}\left(a \Phi^{\prime \prime}+c \Phi^{\prime}\right) .
$$

From the above equation, if we substitute $\ddot{\Psi}$ in (47), we can obtain the following form:

$$
\begin{gathered}
\left(b^{2} r^{2}-a^{2}\right)\left[\frac{\Phi^{\prime} \Psi^{\prime}}{\Phi \Psi}-\frac{\Phi^{\prime \prime}}{\Phi}\right]+\left(2 a^{2}-b^{2} r^{2}\right)\left(\frac{c \Phi^{\prime}}{a \Phi}\right) \\
-a c\left(\frac{\Psi^{\prime}}{\Psi}\right)+c^{2}=0 .
\end{gathered}
$$

Equation (49) is a nonlinear ODE which is very difficult to solve. However, it is worth noting that this equation is easy to solve when $a=b r$. In this case, we can integrate (49) and obtain the following:

$$
\Phi(\xi)=a_{1} \Psi(\xi) \exp \left[-\frac{c \xi}{b r}\right]
$$

where $a_{1}$ is an arbitrary constant of integration. Substituting (50) in (46), we have the following ODE of function $\Psi$ only:

$$
2 b r \Psi^{\prime \prime}=c \Psi .
$$

The general solution of the above equation is

$$
\Psi(\xi)=a_{3}+a_{2} \exp \left[\frac{c \xi}{2 b r}\right],
$$

where $a_{2}$ and $a_{3}$ are arbitrary constants of integration. Now, by using (50) and (33), we can find the solution as follows:

$$
\begin{aligned}
A(t) & =r, \\
f(x, t) & =a_{3}+a_{2} \exp \left[\frac{c(t+r x)}{2 b r}\right], \\
g(x, t) & =a_{1} \exp \left[-\frac{c t}{r}\right]\left(a_{3}+a_{2} \exp \left[\frac{c(t+r x)}{2 b r}\right]\right) .
\end{aligned}
$$

It is observed from (53) and (3) that the line element (2) can be written in the following form:

$$
\begin{aligned}
d s^{2}= & d t^{2}-r^{2} d x^{2}-r^{2}\left(a_{3}+a_{2} \exp \left[\frac{c(t+r x)}{2 b r}\right]\right)^{2} \\
& \times\left(d y^{2}+a_{1}^{2} \exp \left[-\frac{2 c t}{r}\right] d z^{2}\right)
\end{aligned}
$$

where $r, c, b, a_{1}, a_{2}$, and $a_{3}$ are arbitrary constants. 


\section{Physical Properties of the Model}

The field equations (15)-(16) constitute a system of five highly nonlinear differential equations with seven unknowns variables, $A, f, g, p, \rho, \bar{\mu}$, and $\beta$. The symmetries give one condition (24) for function $A$. Therefore, one physically reasonable condition amongst these parameters is required to obtain explicit solutions of the field equations. Let us assume that the density $\rho$ and the pressure $p$ are related by barotropic equation of state:

$$
p=\lambda \rho, \quad 0 \leq \lambda \leq 1
$$

5.1. For Model (44). Using (43) in the Einstein field equations (16), with taking into account condition (55), the expressions for density $\rho$, pressure $p$, magnetic permeability $\bar{\mu}$, and displacement field $\beta$ are given by

$$
\begin{aligned}
& \rho(x, t)= \frac{9}{5 \chi(1-\lambda)} \\
& \times\left[\frac{5 a_{2}+[\exp [a x] /(t+b)]^{3 / 4}}{(t+b)^{2}\left(a_{2}+[\exp [a x] /(t+b)]^{3 / 4}\right)}\right], \\
& p(x, t)= \frac{9 \lambda}{5 \chi(1-\lambda)} \\
& \times\left[\frac{5 a_{2}+[\exp [a x] /(t+b)]^{3 / 4}}{(t+b)^{2}\left(a_{2}+[\exp [a x] /(t+b)]^{3 / 4}\right)}\right], \\
& \bar{\mu}(x, t)= \frac{\chi F_{12}^{2}(x)}{3 a^{2} a_{2} d^{2}(t+b)^{2}\left(a_{2}+\left[\frac{\exp [a x]}{t+b}\right]^{3 / 4}\right)}, \\
& \beta^{2}(x, t)= \frac{2}{15}\left[\left(5 a_{2}(5+13 \lambda)\right.\right. \\
& \times(\lambda-1)(t+b)^{2} \times\left(a_{2}+\left[\frac{\left.\exp [a x]]^{3 / 4}\right)}{t+b}\right)^{-1}\right] \\
&\left.+2(2+7 \lambda)\left[\frac{\exp [a x]}{t+b}\right]^{3 / 4}\right)
\end{aligned}
$$

For the line element (44), using (4), (8), and (9), we have the following physical properties. The volume element is

$$
V=a d q(t+b)^{4}\left(a_{2}+\left[\frac{\exp [a x]}{t+b}\right]^{3 / 4}\right)^{8 / 5} .
$$

The expansion scalar, which determines the volume behavior of the fluid, is given by

$$
\Theta=\frac{2}{5}\left[\frac{10 a_{2}+7[\exp [a x] /(t+b)]^{3 / 4}}{(t+b)\left(a_{2}+[\exp [a x] /(t+b)]^{3 / 4}\right)}\right] .
$$

The nonvanishing components of the shear tensor, $\sigma_{i}^{j}$, are

$$
\begin{aligned}
& \sigma_{1}^{1}=\frac{[\exp [a x] /(t+b)]^{3 / 4}-5 a_{2}}{15(t+b)\left(a_{2}+[\exp [a x] /(t+b)]^{3 / 4}\right)}, \\
& \sigma_{2}^{2}=-\frac{1}{30}\left[\frac{10 a_{2}+[\exp [a x] /(t+b)]^{3 / 4}}{(t+b)\left(a_{2}+[\exp [a x] /(t+b)]^{3 / 4}\right)}\right], \\
& \sigma_{3}^{3}=\frac{4 a_{2}+7[\exp [a x] /(t+b)]^{3 / 4}}{6(t+b)\left(a_{2}+[\exp [a x] /(t+b)]^{3 / 4}\right)}, \\
& \sigma_{4}^{4}=-\frac{4}{15}\left[\frac{10 a_{2}+7[\exp [a x] /(t+b)]^{3 / 4}}{(t+b)\left(a_{2}+[\exp [a x] /(t+b)]^{3 / 4}\right)}\right] .
\end{aligned}
$$

Hence the shear scalar $\sigma$ is given by

$$
\begin{aligned}
\sigma^{2}= & \left(3500 a_{2}^{2}+4630 a_{2}[\exp [a x] /(t+b)]^{3 / 4}\right. \\
& \left.+1607[\exp [a x] /(t+b)]^{3 / 2}\right) \\
\times & \left(900(t+b)^{2}\left(a_{2}+[\exp [a x] /(t+b)]^{3 / 4}\right)^{2}\right)^{-1} .
\end{aligned}
$$

The model does not admit acceleration and rotation, since $\dot{u}_{i}=0$ and $\omega_{i j}=0$. We can see that

$$
\frac{\sigma_{4}^{4}}{\Theta}=-\frac{2}{3}
$$

which is a constant of proportional. We found also that

$$
\lim _{t \rightarrow \infty} \frac{\sigma^{2}}{\Theta}=\frac{5 \sqrt{35}}{2} \neq 0
$$

and this means that there is no possibility that the universe may got isotropized in some later time; that is, it remains anisotropic for all times.

5.2. For Model (54). Using (53) in the Einstein field equations (16), with taking into account condition (55), the expressions 
for density $\rho$, pressure $p$, magnetic permeability $\bar{\mu}$, and displacement field $\beta$ are given by

$$
\begin{aligned}
\rho(x, t)= & \frac{c^{2}}{\chi r^{2}(\lambda-1)}\left(\frac{c_{2} \exp [c(t+r x) / 2 r]-c_{3}}{c_{2} \exp [c(t+r x) / 2 r]+c_{3}}\right), \\
p(x, t)= & \frac{\lambda c^{2}}{\chi r^{2}(\lambda-1)}\left(\frac{c_{2} \exp [c(t+r x) / 2 r]-c_{3}}{c_{2} \exp [c(t+r x) / 2 r]+c_{3}}\right), \\
\bar{\mu}(x, t)= & \frac{\chi F_{12}^{2}(x)}{c_{3} c^{2} r^{2}}\left(c_{2} \exp \left[\frac{c(t+r x)}{2 r}\right]+c_{3}\right)^{-1}, \\
\beta^{2}(x, t)= & \frac{2 c^{2}}{3 r^{2}(\lambda-1)} \\
& \times\left(\frac{c_{3}(1+\lambda)-2 \lambda c_{2} \exp [c(t+r x) / 2 r]}{c_{3}+c_{2} \exp [c(t+r x) / 2 r]}\right) .
\end{aligned}
$$

For the line element (54), using (4), (8), and (9), we have the following physical properties. The volume element is

$$
V=c_{1} r^{3} \exp \left[-\frac{c t}{r}\right]\left(c_{3}+c_{2} \exp \left[\frac{c(t+r x)}{2 r}\right]\right)^{2} .
$$

The expansion scalar, which determines the volume behavior of the fluid, is given by

$$
\Theta=-\frac{c c_{3}}{r}\left(c_{3}+c_{2} \exp \left[\frac{c(t+r x)}{2 r}\right]\right)^{-1} .
$$

The nonvanishing components of the shear tensor, $\sigma_{i}^{j}$, satisfy

$$
\begin{gathered}
\frac{\sigma_{1}^{1}}{\Theta}=-\frac{1}{3} \\
\frac{\sigma_{2}^{2}}{\Theta}=-\left(\frac{1}{3}+\frac{c_{2}}{2 c_{3}} \exp \left[\frac{c(t+r x)}{2 r}\right]\right), \\
\frac{\sigma_{3}^{3}}{\Theta}=\frac{2}{3}+\frac{c_{2}}{2 c_{3}} \exp \left[\frac{c(t+r x)}{2 r}\right], \\
\frac{\sigma_{4}^{4}}{\Theta}=-\frac{2}{3} .
\end{gathered}
$$

Hence the shear scalar $\sigma$ is given by

$$
\sigma^{2}=\frac{c^{2}}{4 r^{2}}+\frac{11 c_{3}^{2} c^{2}}{36 r^{2}}\left(c_{3}+c_{2} \exp \left[\frac{c(t+r x)}{2 r}\right]\right)^{-2}
$$

The model does not admit acceleration and rotation, since $\dot{u}_{i}=0$ and $\omega_{i j}=0$.

\section{Conflict of Interests}

The author declares that there is no conflict of interests regarding the publication of this paper.

\section{Acknowledgment}

This paper was funded by the Deanship of Scientific Research (DSR), King Abdulaziz University, Jeddah, under Grant no. 130-016-D1434. The author, therefore, acknowledges with thanks DSR technical and financial support.

\section{References}

[1] A. Krasinski, Inhomogeneous Cosmological Models, Cambridge University Press, Cambridge, UK, 1997.

[2] G. Lyra, "Über eine Modifikation der Riemannschen Geometrie," Mathematische Zeitschrift, vol. 54, pp. 52-64, 1951.

[3] D. K. Sen, "A static cosmological model," Zeitschrift für Physik C: Particles and Fields, vol. 149, pp. 311-323, 1957.

[4] D. K. Sen, "On geodesics of a modified Riemannian manifold," Canadian Mathematical Society, vol. 3, pp. 255-261, 1960.

[5] D. K. Sen and K. A. Dunn, "A scalar-tensor theory of gravitation in a modified Riemannian manifold," Journal of Mathematical Physics, vol. 12, pp. 578-586, 1971.

[6] W. D. Halford, "Cosmological theory based on Lyra's geometry," Australian Journal of Physics, vol. 23, no. 5, pp. 863-870, 1970.

[7] S. S. De and F. Rahaman, Finsler Geometry of Hadrons and Lyra Geometry: Cosmological Aspects, Lambert Academic Publishing, Saarbrücken, Germany, 2012.

[8] A. Pradhan, I. Aotemshi, and G. P. Singh, "Plane symmetric domain wall in Lyra geometry," Astrophysics and Space Science, vol. 288, no. 3, pp. 315-325, 2003.

[9] A. Pradhan and A. K. Vishwakarma, "A new class of LRS Bianchi type-I cosmological models in Lyra geometry," Journal of Geometry and Physics, vol. 49, no. 3-4, pp. 332-342, 2004.

[10] A. Pradhan and S. S. Kumhar, "Plane symmetric inhomogeneous perfect fluid universe with electromagnetic field in Lyra geometry," Astrophysics and Space Science, vol. 321, no. 2, pp. 137-146, 2009.

[11] A. Pradhan and P. Ram, "A plane-symmetric magnetized inhomogeneous cosmological models of perfect fluid distribution with variable magnetic permeability in Lyra geometry," International Journal of Theoretical Physics, vol. 48, no. 11, pp. 3188-3201, 2009.

[12] A. Pradhan, H. Amirhashchi, and H. Zainuddin, "A new class of inhomogeneous cosmological models with electromagnetic field in normal gauge for Lyra's manifold," International Journal of Theoretical Physics, vol. 50, no. 1, pp. 56-69, 2011.

[13] P. Anirudh, A. Singh, and R. S. Singh, "A plane-symmetric magnetized inhomogeneous cosmological model of perfect fluid distribution with variable magnetic permeability," Romanian Reports of Physics, vol. 56, no. 1-2, pp. 297-307, 2011.

[14] A. Pradhan and A. K. Singh, "Anisotropic Bianchi type-I string cosmological models in normal Gauge for Lyra's manifold with constant deceleration parameter," International Journal of Theoretical Physics, vol. 50, no. 3, pp. 916-933, 2011.

[15] R. Casana, C. A. M. de Melo, and B. M. Pimentel, "Spinorial field and Lyra geometry," Astrophysics and Space Science, vol. 305, no. 2, pp. 125-132, 2006.

[16] F. Rahaman, B. C. Bhui, and G. Bag, "Can Lyra geometry explain the singularity free as well as accelerating Universe?" Astrophysics and Space Science, vol. 295, no. 4, pp. 507-513, 2005.

[17] R. Bali and N. K. Chandnani, "Bianchi type-I cosmological model for perfect fluid distribution in Lyra geometry," Journal of 
Mathematical Physics, vol. 49, no. 3, Article ID 032502, 8 pages, 2008.

[18] R. Bali and N. K. Chandnani, "Bianchi type V barotropic perfect fluid cosmological model in Lyra geometry," International Journal of Theoretical Physics, vol. 48, no. 5, pp. 1523-1533, 2009.

[19] S. Kumar and C. P. Singh, "An exact Bianchi type-I cosmological model in Lyra's manifold," International Journal of Modern Physics A, vol. 23, no. 6, pp. 813-822, 2008.

[20] A. K. Yadav, A. Pradhan, and A. Singh, "LRS Bianchi typeII massive string cosmological models in general relativity," Romanian Reports of Physics, vol. 56, no. 7-8, pp. 1019-1034, 2011.

[21] V. U. M. Rao, T. Vinutha, and M. V. Santhi, "Bianchi type-V cosmological model with perfect fluid using negative constant deceleration parameter in a scalar tensor theory based on Lyra Manifold," Astrophysics and Space Science, vol. 314, no. 1-3, pp. 213-216, 2008.

[22] R. Zia and R. P. Singh, "Bulk viscous inhomogeneous cosmological models with electromagnetic field in Lyra geometry," Romanian Journal of Physics, vol. 57, no. 3-4, pp. 761-778, 2012.

[23] A. T. Ali and F. Rahaman, "New class of magnetized inhomogeneous Bianchi type-I cosmological model with variable magnetic permeability in Lyra geometry," International Journal of Theoretical Physics, vol. 52, no. 11, pp. 4055-4067, 2013.

[24] A. T. Ali, "New generalized Jacobi elliptic function rational expansion method," Journal of Computational and Applied Mathematics, vol. 235, no. 14, pp. 4117-4127, 2011.

[25] A. T. Ali and E. R. Hassan, "General Expa-function method for nonlinear evolution equations," Applied Mathematics and Computation, vol. 217, no. 2, pp. 451-459, 2010.

[26] M. F. El-Sabbagh and A. T. Ali, "New exact solutions for $(3+1)$ dimensional Kadomtsev-Petviashvili equation and generalized $(2+1)$-dimensional Boussinesq equation," International Journal of Nonlinear Sciences and Numerical Simulation, vol. 6, no. 2, pp. 151-162, 2005.

[27] M. F. El-Sabbagh and A. T. Ali, "New generalized Jacobi elliptic function expansion method," Communications in Nonlinear Science and Numerical Simulation, vol. 13, no. 9, pp. 1758-1766, 2008.

[28] L. V. Ovsiannikov, Group Analysis of Differential Equations, translated by Y. Chapovsky, edited by W. F. Ames, Academic Press, New York, NY, USA, 1982.

[29] G. W. Bluman and S. Kumei, Symmetries and Differential Equations in Applied Sciences, Springer, New York, NY, USA, 1989.

[30] N. H. Ibragimov, Transformation Groups Applied to Mathematical Physics, D. Reidel, Dordrecht, The Netherlands, 1985.

[31] P. J. Olver, Applications of Lie Groups to Differential Equations, vol. 107 of Graduate Texts in Mathematics, Springer, New York, NY, USA, 2nd edition, 1993.

[32] A. T. Ali, A. K. Yadav, and S. R. Mahmoud, "Some plane symmetric inhomogeneous cosmological models in the scalartensor theory of gravitation," Astrophysics and Space Science, vol. 349, no. 1, pp. 539-547, 2014.

[33] A. T. Ali, "New exact solutions of the Einstein vacuum equations for rotating axially symmetric fields," Physica Scripta, vol. 79, no. 3, Article ID 035006, 2009.

[34] S. K. Attallah, M. F. El-Sabbagh, and A. T. Ali, "Isovector fields and similarity solutions of Einstein vacuum equations for rotating fields," Communications in Nonlinear Science and Numerical Simulation, vol. 12, no. 7, pp. 1153-1161, 2007.
[35] K. S. Mekheimer, S. Z. Husseny, A. T. Ali, and A. E. Abo-Elkhair, "Lie point symmetries and similarity solutions for an electrically conducting Jeffrey fluid," Physica Scripta, vol. 83, no. 1, Article ID 015017, 2011.

[36] A. K. Raychaudhuri, Theoritical Cosmology, Oxford Science Publications, Oxford, UK, 1979.

[37] A. Lichnerowicz, Relativistic Hydrodynamics and MagnetoHydro-Dynamics, W. A. Benjamin, New York, NY, USA, 1967.

[38] A. Pradhan and P. Mathur, "Inhomogeneous perfect fluid universe with electromagnetic field in Lyra geometry," Fizika B, vol. 18, no. 4, pp. 243-264, 2009. 


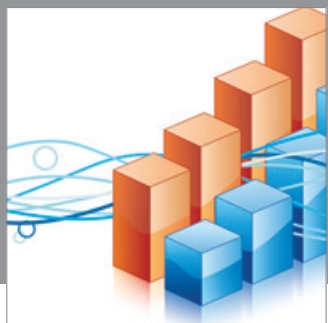

Advances in

Operations Research

mansans

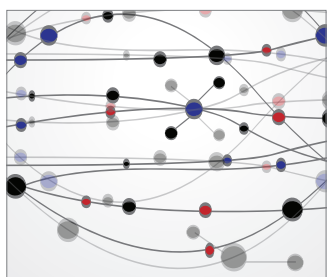

The Scientific World Journal
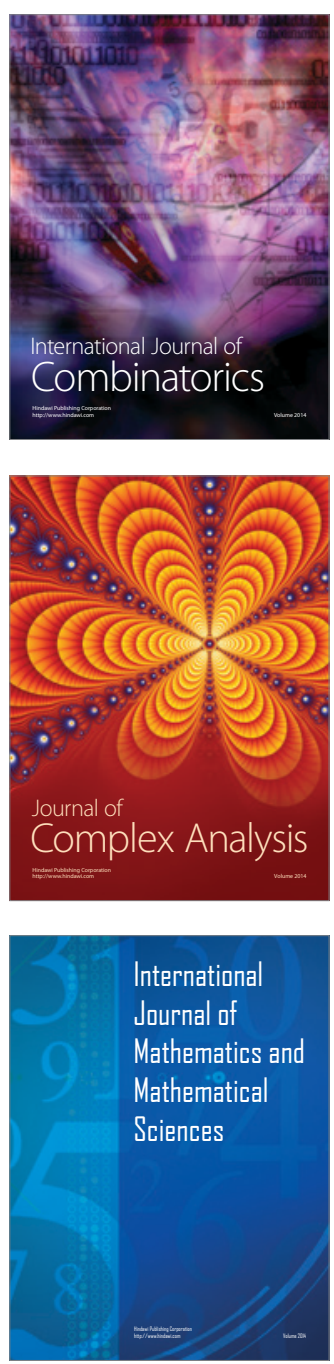
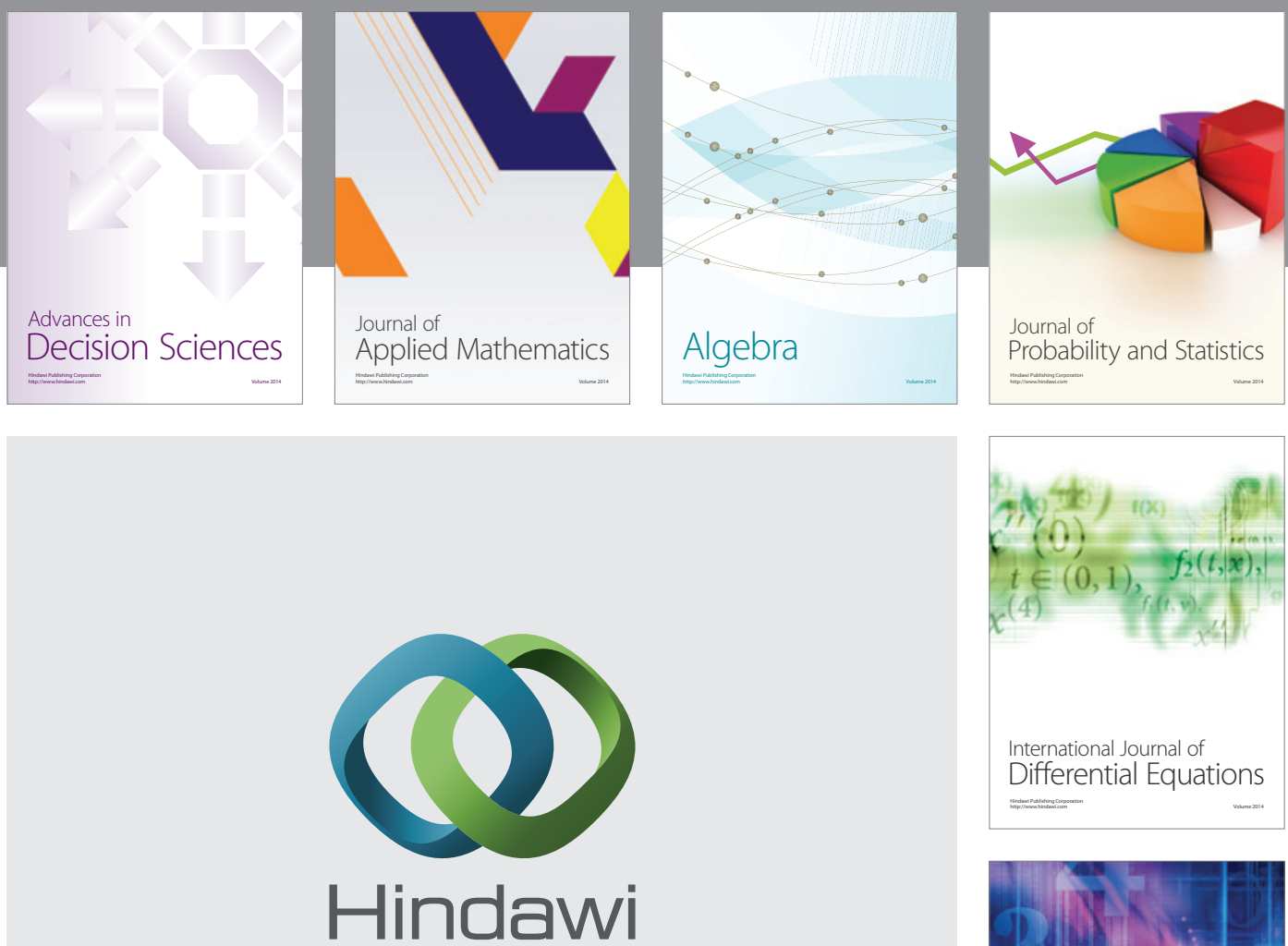

Submit your manuscripts at http://www.hindawi.com
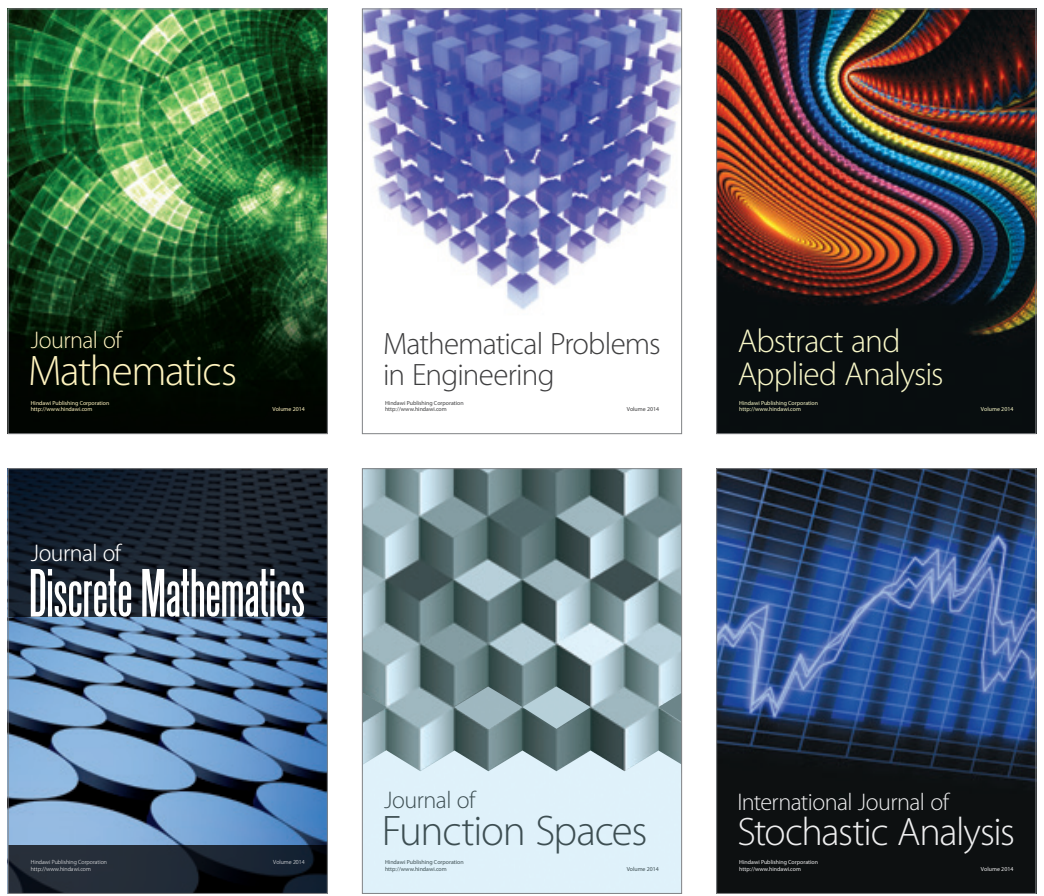

Journal of

Function Spaces

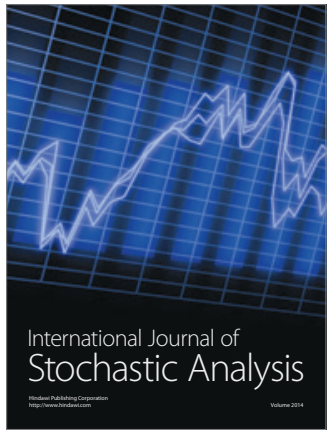

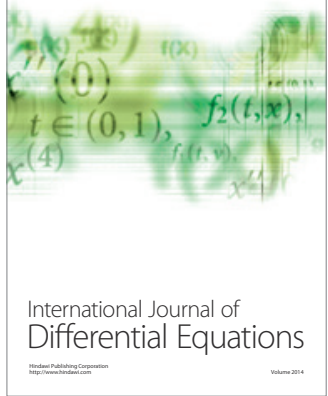
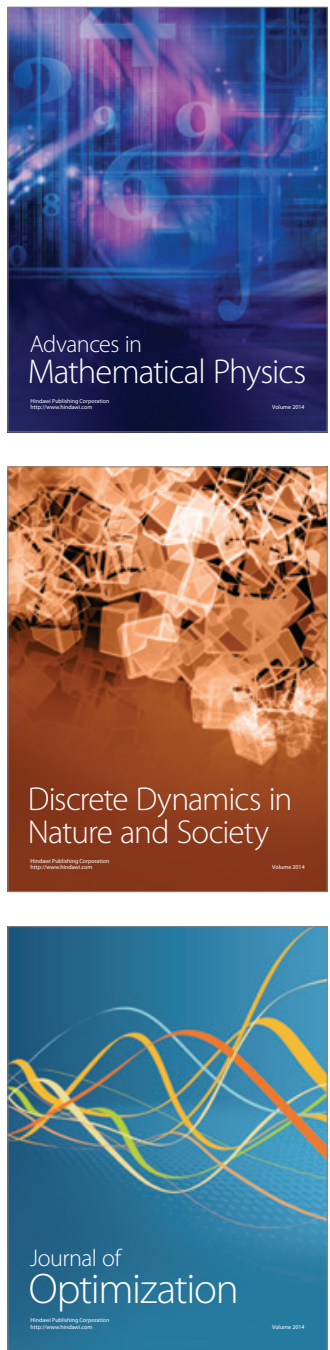